\title{
Circulating biomarkers of nitric oxide bioactivity and impaired muscle vasoreactivity to exercise in adults with uncomplicated type 1 diabetes
}

\author{
Elodie Lespagnol ${ }^{1}$ (D) Sémah Tagougui $^{1}$ (D) $\cdot$ Bernadette O. Fernandez ${ }^{2}$ (D) $\cdot$ Farid Zerimech $^{3}$ (D) Régis Matran $^{3}$ (D) \\ Patrice Maboudou $^{4}$ - Serge Berthoin ${ }^{1}$ (D) Amandine Descat $^{5}$ (D) $\cdot$ Isabelle Kim ${ }^{4} \cdot$ Mehdi Pawlak-Chaouch $^{1}$ (I) . \\ Julien Boissière ${ }^{1}$ (D) $\cdot$ Eric Boulanger $^{6}$ (D) $\cdot$ Martin Feelisch $^{2}$ (D) $\cdot$ Pierre Fontaine $^{7}$ (D) $\cdot$ Elsa Heyman $^{1}$ (D)
}

Received: 28 July 2020 / Accepted: 29 September 2020 / Published online: 21 November 2020

(C) Springer-Verlag GmbH Germany, part of Springer Nature 2020

\begin{abstract}
Aims/hypothesis Early compromised endothelial function challenges the ability of individuals with type 1 diabetes to perform normal physical exercise. The exact mechanisms underlying this vascular limitation remain unknown, but may involve either formation or metabolism of nitric oxide (NO), a major vasodilator, whose activity is known to be compromised by oxidative stress.

Methods Muscle microvascular reactivity (near-infrared spectroscopy) to an incremental exhaustive bout of exercise was assessed in 22 adults with uncomplicated type 1 diabetes $\left(\mathrm{HbA}_{1 \mathrm{c}} 64.5 \pm 15.7 \mathrm{mmol} / \mathrm{mol} ; 8.0 \pm 1.4 \%\right)$ and in 21 healthy individuals (18-40 years of age). NO-related substrates/metabolites were also measured in the blood along with other vasoactive compounds and oxidative stress markers; measurements were taken at rest, at peak exercise and after 15 min of recovery. Demographic characteristics, body composition, smoking status and diet were comparable in both groups.

Results Maximal oxygen uptake was impaired in individuals with type 1 diabetes compared with in healthy participants $(35.6 \pm$ 7.7 vs $39.6 \pm 6.8 \mathrm{ml} \mathrm{min}^{-1} \mathrm{~kg}^{-1}, p<0.01$ ) despite comparable levels of habitual physical activity (moderate to vigorous physical activity by accelerometery, $234.9 \pm 160.0$ vs $280.1 \pm 114.9 \mathrm{~min} /$ week). Compared with non-diabetic participants, individuals with type 1 diabetes also displayed a blunted exercise-induced vasoreactivity (muscle blood volume at peak exercise as reflected by $\Delta$ total haemoglobin, $2.03 \pm 5.82$ vs $5.33 \pm 5.54 \mu \mathrm{mol} / 1$; interaction 'exercise' $\times$ 'group', $p<0.05)$; this was accompanied by lower $\mathrm{K}^{+}$concentration $(p<0.05)$, reduced plasma L-arginine $(p<0.05)$ - in particular when $\mathrm{HbA}_{1 \mathrm{c}}$ was high (mean estimation: $-4.0, p<0.05)$ - and lower plasma urate levels $(p<0.01)$. Nonetheless, exhaustive exercise did not worsen lipid peroxidation or other oxidative stress biomarkers, and erythrocytic enzymatic antioxidant resources were mobilised to a comparable extent in both groups. Nitrite and total nitrosation products, which are potential alternative NO sources, were similarly unaltered.
\end{abstract}

Supplementary Information The online version contains peer-reviewed but unedited supplementary material available at https://doi.org/10.1007/ s00125-020-05329-8.

Elsa Heyman

elsa.heyman@univ-lille.fr

1 ULR 7369 - URePSSS - Unité de Recherche Pluridisciplinaire Sport Santé Société, Université Lille, Université Artois, Université Littoral Côte d'Opale, Lille, France

2 Clinical \& Experimental Sciences, Faculty of Medicine, University of Southampton and University Hospital Southampton NHS Foundation Trust, Southampton, UK

3 CHU Lille, Institut Pasteur de Lille, ULR 4483 - IMPECS - IMPact de l'Environnement Chimique sur la Santé humaine, Université Lille, Lille, France
4 CHU de Lille, Laboratoire de Biochimie et Biologie Moléculaire, Pôle de Biologie Pathologie Génétique, Lille, France

5 CHU Lille, ULR 7365 - GRITA - Groupe de Recherche sur les formes Injectables et les Technologies Associées, Université Lille, Lille, France

6 Inserm, CHU Lille, Pasteur Institute of Lille, U1167 - RID-AGE, Université Lille, Lille, France

7 Department of Diabetology, Lille University Hospital, EA 4489 Lille, France 


\section{Research in context}

\section{What is already known about this subject?}

- Defects in exercise-induced microvascular reactivity and reduced aerobic fitness appear before overt vascular complications in type 1 diabetes

- In non-diabetic individuals, nitric oxide (NO•) is recognised as an important contributor to exercise-induced vasodilation, since it is enhanced by shear stress-induced activation of endothelial NO. synthase (eNOS) and by alternative sources (e.g. nitrite) under hypoxic conditions

- Limited data are available about NO metabolism during exercise in type 1 diabetes, while diabetes-associated oxidative stress can compromise $\mathrm{NO} \cdot$ bioavailability

What is the key question?

- Is the impaired muscle vasoreactivity to exercise in type 1 diabetes accompanied by defects in the ability to generate NO- and by oxidative stress exacerbation?

What are the new findings?

- In individuals with uncomplicated type 1 diabetes, plasma L-arginine (the essential substrate for eNOS) and plasma urate (a major water-soluble antioxidant) are reduced

- However, nitrite and total nitrosation products are unaltered and exhaustive exercise does not worsen lipid peroxidation

\section{How might this impact on clinical practice in the foreseeable future?}

- Considering L-arginine depletion among people with type 1 diabetes, supplementation with L-citrulline (naturally found in some fruits and nuts) might be relevant for eNOS-dependent vasodilatation, particularly for people with an active lifestyle

Conclusions/interpretation Participants with uncomplicated type 1 diabetes displayed reduced availability of L-arginine, the essential substrate for enzymatic nitric oxide synthesis, as well as lower levels of the major plasma antioxidant, urate. Lower urate levels may reflect a defect in the activity of xanthine oxidase, an enzyme capable of producing NO from nitrite under hypoxic conditions. Thus, both canonical and non-canonical NO production may be reduced. However, neither of these changes exacerbated exercise-induced oxidative stress.

Trial registration clinicaltrials.gov NCT02051504

Keywords Aerobic fitness · Antioxidant defences $\cdot$ L-arginine $\cdot$ Nitric oxide $\cdot$ Oxidative stress $\cdot$ Physical exercise $\cdot$ Skeletal muscle $\cdot$ Type 1 diabetes $\cdot$ Urate $\cdot$ Vasoreactivity

$\begin{array}{ll}\text { Abbreviations } & \\ \text { 8-iso-PGF2 } \alpha & \text { 8-iso-Prostaglandin- } \mathrm{F}_{2 \alpha} \\ \text { ADMA } & \text { Asymmetric dimethylarginine } \\ \mathrm{CML} & N \text {-Carboxymethyl lysine } \\ \mathrm{DEXA} & \text { Dual-energy x-ray absorptiometry } \\ \text { eNOS } & \text { Endothelial nitric oxide synthase } \\ \mathrm{HHb} & \text { Deoxyhaemoglobin } \\ \mathrm{NIRS} & \text { Near-infrared spectroscopy } \\ \mathrm{NO} \bullet & \text { Nitric oxide } \\ \mathrm{NOS} & \text { Nitric oxide synthase } \\ \mathrm{PaO} & \text { Partial pressure of } \mathrm{O}_{2} \text { in arterial blood } \\ \mathrm{PaCO}_{2} & \text { Partial pressure of } \mathrm{CO}_{2} \text { in arterial blood } \\ \mathrm{RXNO}_{\mathrm{SDMA}} & \text { Total nitrosation products } \\ & \text { Symmetric dimethylarginine }\end{array}$

SOD Superoxide dismutase

$\mathrm{THb} \quad$ Total haemoglobin

\section{Introduction}

Endothelial dysfunction [1] is a main risk factor for the development of micro- and/or macrovascular complications in individuals with type 1 diabetes [2]. A recent meta-analysis highlighted the presence of early endothelial dysfunction in these patients, without overt vascular complications [3]. This dysfunction can even appear in response to day-to-day whole- 
body aerobic exercise, specifically at the level of microvessels close to active skeletal muscle [4-6]. Understanding the mechanisms involved in blunted muscle microvascular reactivity to exercise in uncomplicated type 1 diabetes is of great clinical relevance. As such, in addition to possible long-term effects on primary determinants of diabetes complications, impaired blood supply to skeletal muscle may be involved in lower aerobic fitness [4-6], a strong predictor of cardiovascular mortality [7].

The exact mechanisms underlying type 1 diabetesassociated vascular limitation to exercise remain unknown, but could conceivably involve either formation or metabolism of nitric oxide (NO•) [8]. Exercise-induced dilatation of terminal arterioles increases blood flow to the muscle and increases shear stress in upstream resistance vessels. The latter stimulates endothelial NO• synthesis from the semi-essential amino acid L-arginine through the action of endothelial nitric oxide synthase (eNOS). $\mathrm{NO} \bullet$ then rapidly diffuses into the vascular smooth muscle where it induces relaxation. Alternatively, bioactive NO• can be formed by non-canonical pathways involving nitrite, especially under acidic and/or hypoxemic conditions, in active skeletal muscle during exercise [9].

Limited data are available about $\mathrm{NO} \cdot$ metabolism in response to acute exercise in type 1 diabetes. Fayh et al investigated whether $\mathrm{NO} \bullet$ production was unaltered in adults with uncomplicated type 1 diabetes after $45 \mathrm{~min}$ of exercise; they measured plasma levels of NOx (i.e. the sum of nitrite and nitrate) using methods that did not distinguish nitrite from nitrate [10]. However, this result requires further validation because only nitrite accurately reflects acute changes in eNOS activity [9]. A study by Fujii et al, using local administration of a non-selective nitric oxide synthase (NOS) inhibitor, suggested no differences in NOS-dependent cutaneous vasodilation between young athletes with type 1 diabetes and healthy individuals during two 30-min bouts of moderate cycling exercise [11]. However, the focus on cutaneous vasculature and the unusual environmental conditions (temperatures of $35^{\circ} \mathrm{C}$ ) limit extrapolation of their findings to normal daily conditions.

Our previous results suggest the involvement of chronic hyperglycaemia in impaired muscle vasoreactivity to intense exercise in uncomplicated type 1 diabetes [6]. Hyperglycaemia increases superoxide anion production through various processes, including excess leakage of electrons from the mitochondrial electron chain, activation of protein kinase $\mathrm{C}$ and hence membrane-associated NAD $(\mathrm{P}) \mathrm{H}-$ dependent oxidases, and increase in intracellular advanced glycation end-products. Superoxide anions can react with NO• to form the powerful pro-oxidant, peroxynitrite [2]. The latter causes peroxidation of lipids and nitr(os)ation of tyrosine moieties and can also uncouple eNOS, which then preferentially increases superoxide anion production over $\mathrm{NO}$ • production. Physical exercise can increase the oxygen flow in skeletal muscle by a factor of 100-200 while exacerbating the production of superoxide. In view of the complexity of the underlying regulation, better insight into oxidative stress markers and antioxidant capacities in response to exercise in type 1 diabetes is warranted.

Considering the clinical implications of vascular limitations to physical exercise in people with uncomplicated type 1 diabetes and the limited understanding of the underlying mechanisms, we took a combined functional/analytical approach measuring precursors, derivatives, and breakdown products of $\mathrm{NO} \cdot$ as well as oxidative stress-related biomarkers together with physiological measurements of exerciseinduced muscle vasoreactivity. We hypothesised that the ability to generate $\mathrm{NO} \cdot$ in response to exercise is compromised and reactive oxygen species production is higher in people with diabetes than in healthy individuals.

\section{Methods}

After providing written consent, 22 patients aged between 18 and 40 with type 1 diabetes for at least 1 year were included in the study. Ethics approval for this study was obtained from the North-Western IV Regional Ethics Committee, NEudraCT (approval number: 2009-A00746-51). The absence of macrovascular and microvascular complications was carefully assessed by the endocrinologist (retinopathy: fundus exam; nephropathy: microalbuminuria, urinaryserum creatinine; peripheral neuropathy: reflexes, $10 \mathrm{~g}$ monofilament, tuning fork; autonomic neuropathy: neurogenic bladder, response to standing, gastroparesis, enteropathy). Twenty-one healthy participants (normal glucose tolerance checked with OGTT, WHO criteria) were recruited from patients' contacts as a control group. Body composition was measured by dual-energy x-ray absorptiometry (DEXA, HOLOGIC). We also checked that the subcutaneous skinfold thickness was $1.5 \mathrm{~cm}$ at the vastus lateralis to ensure the accuracy of near-infrared spectroscopy (NIRS) measurements. Physical activity level was assessed by accelerometery over 1 week with GT1M ActiGraph and by the Modified Activity Questionnaire [12]. Usual daily macronutrient intake was assessed via a 3 day diary ( 2 weekdays and 1 weekend day), then checked and supplemented by a research-trained dietitian during a face to face appointment, with special focus on nitrite, nitrate, polyphenol and antioxidant consumption.

Participants came to the laboratory twice, and refrained from vigorous activity for $48 \mathrm{~h}$ beforehand and from smoking on the morning of each visit. For the first visit, participants ate a standardised breakfast (electronic supplementary material [ESM] Table 1) and individuals with type 1 diabetes received their usual insulin dose. A maximal graded test on a cycle 
ergometer was performed (Excalibur, Lode; 2 min resting period, first stage at $30 \mathrm{~W}$ and then $20 \mathrm{~W} / 2 \mathrm{~min}$ increments until exhaustion; at an ambient temperature of $18-20^{\circ} \mathrm{C}$ ), 3.4 $\pm 0.6 \mathrm{~h}$ after the breakfast. Throughout exercise, ECG and pulmonary gas exchanges were monitored (breath-by-breath system, Ergocard). $\dot{V} \mathrm{O}_{2 \max }$ was recorded as the highest $15 \mathrm{~s}$ mean value at test termination and was validated for all the participants (i.e. three of the five Astrand criteria observed). No hypoglycaemic episode occurred during maximal exercise.

Muscle haemodynamic response was monitored continuously throughout exercise. Postprandial venous and arterialised blood samples were collected at rest and immediately after completion of maximal graded exercise (i.e. following peak exercise); an additional venous blood sample was taken 15 min into the passive recovery phase. During the second visit, after an $8 \mathrm{~h}$ overnight fast (ESM Table 1), an additional venous blood sample was obtained.

Exercise-induced muscle vasoreactivity A $4 \mathrm{~cm}$-interspersed NIRS (Oxymon MkIII, Artinis) emitter-detector optode was placed on the right vastus lateralis muscle and light absorption (two continuous wavelengths, 780 and $850 \mathrm{~nm}$ ) was monitored at a $10 \mathrm{~Hz}$ frequency. The Beer-Lambert law and a normalisation against the baseline period (i.e. 2 min resting, sitting on the cycle ergometer) were used to determine the exercise-induced changes in muscle microvessel blood volume ( $\Delta$ total haemoglobin $[\mathrm{THb}]$, as the arithmetic sum of $\Delta$ oxyhaemoglobin and $\Delta$ deoxyhaemoglobin [HHb]) [13].

Blood analyses Possible underlying mechanisms of NO•dependent vascular function examined in plasma, serum or erythrocyte included: (1) NO• bioavailability (nitrite, nitrate and total nitrosation products [RXNO]); (2) substrates (L-arginine, and with a lower affinity, L-homoarginine) and derivative (L-citrulline) of $\mathrm{NO} \cdot$ synthesis; (3) factors limiting NO• production (the endogenous NOS inhibitor, asymmetric dimethylarginine [ADMA] and the inhibitor of cellular uptake of arginine, symmetric dimethylarginine [SDMA]); and/or (4) factors exacerbating its elimination, i.e. free radical accumulation, as reflected by antioxidant availability (total antioxidant status as well as specific water-soluble [vitamin C, urate, total free thiols] and lipophilic [vitamins A and E] antioxidants; enzymatic erythrocyte defences including superoxide dismutase [SOD], glutathione peroxidase and glutathione reductase activities) and oxidative stress biomarkers (markers of lipid peroxidation, 8-iso-prostaglandin-F2 $\alpha$ [8-iso-PGF2 $\alpha$, equivalent to $15-\mathrm{F}_{2 \mathrm{t}}$-isoprostane] and malondialdehyde; an advanced 'glycoxidation' end-product, $N$-carboxymethyl lysine [CML]; and a marker of the accumulation of protein oxidation products, advanced glycation end-products and advanced lipoxidation end-products, i.e. fluorescent oxidation products).

Note that concentrations of plasma free thiols were normalised to plasma protein to evaluate the plasma reducing capacity for a stable quantity of protein. As well, to get a better insight into the effects of type 1 diabetes and exercise on plasma total antioxidant capacity, this outcome was corrected by urate, which is the most abundant water-soluble antioxidant in blood.

In addition, we considered metabolic data (glucose, nonesterified fatty acid [NEFA], glycerol), other vasoactive moieties (including catecholamines, adiponectin, leptin, free insulin $[2,14]$ ), glucagon (which can modulate amino acid concentrations, including L-arginine and L-citrulline [15]), and in arterialised blood, $\mathrm{K}^{+}$, lactate and partial pressure of $\mathrm{CO}_{2}\left(\mathrm{PaCO}_{2}\right)$. We also assessed factors favouring $\mathrm{NO} \bullet$ release from circulating $S$-nitrosylated blood proteins or nitrite (i.e. decrease in partial pressure of $\mathrm{O}_{2}$ in arterial blood $\left[\mathrm{PaO}_{2}\right]$ and $\mathrm{pH}$, increase in oxyhaemoglobin dissociation rate, i.e. $\Delta \mathrm{HHb}$, as assessed by NIRS), factors influencing redistribution of nitrite and nitrate between erythrocytes and plasma $\left(\mathrm{PaCO}_{2}\right.$, arterialised blood $\left.\mathrm{HCO}_{3}{ }^{-}\right)$, and a by-product of $\mathrm{NO} \bullet$ oxidation in nitrate (arterialised blood methaemoglobin; $\mathrm{MetHb}$ ). The fasting lipid profile was also measured (Table 1).

The assays used for all the analyses in plasma, serum, erythrocytes, blood, and arterialised blood are detailed in ESM Table 2.

Blood processing Fasting and postprandial (from a forearm catheter) venous and postprandial arterialised blood samples were collected. Haemoglobin and haematocrit were determined on heparin venous blood samples and were used to correct exercise-induced changes in postprandial serum or plasma concentrations (i.e. values at peak exercise and after 15 min of recovery) according to serum or plasma volume decrease (haemoconcentration) [16]. All venous blood samples were then centrifuged (immediately at $855 \mathrm{~g}, 4^{\circ} \mathrm{C}$, 5 min for containers with anticoagulants-EDTA, EDTA plus aprotinin, heparin or fluorinated - and after $1 \mathrm{~h}$ at ambient temperature at $1520 \mathrm{~g}, 4^{\circ} \mathrm{C}, 13 \mathrm{~min}$ for free-additive containers) and resulting supernatant was removed and frozen $\left(-80^{\circ} \mathrm{C}\right)$ pending duplicate future analyses of all the above plasma or serum molecules, except for vitamin $\mathrm{C}$ (vacutainer protected from light), glucose, NEFA, glycerol and glucagon, which were assayed immediately on the day of experimentation. The remaining red blood cells from heparinised vacutainers were washed three times in $\mathrm{NaCl} 0.15 \mathrm{M}$ and finally diluted by addition of ice-cold distilled water (1:1) mixed with vortex and stored at $-80^{\circ} \mathrm{C}$ until duplicate analysis of erythrocyte activities of SOD, GPx and GR. In addition, during the first (postprandial) visit only, at rest and immediately after exercise, a microcapillary arterialised ear-lobe (vasodilatory pomade applied 5 min before) blood sample 
Table 1 Participants' characteristics

\begin{tabular}{|c|c|c|}
\hline & Participants with type 1 diabetes & Healthy participants \\
\hline \multicolumn{3}{|l|}{ Anthropometric and demographic data } \\
\hline$N$ (Male/female sex) & $22(15 / 7)$ & $21(14 / 7)$ \\
\hline Age (years) & $27.3 \pm 7.0(18-39)$ & $27.1 \pm 5.8(19-40)$ \\
\hline BMI (kg.m $\left.{ }^{-2}\right)$ & $23.0 \pm 2.5(17-28)$ & $23.0 \pm 2.4(20-30)$ \\
\hline Fat mass $(\%)$ & $19.8 \pm 6.5(10.7-31.8)$ & $19.0 \pm 5.8(9.1-28.7)$ \\
\hline Fat mass of right leg $(\%)$ & $23.3 \pm 8.2(13.0-38.0)$ & $22.4 \pm 8.1(10.0-38.0)$ \\
\hline Smoking status (smoker/non-smoker) & $5 / 17$ & $6 / 15$ \\
\hline Diabetes duration (years) & $8.7 \pm 5.4(1.0-19.7)$ & NA \\
\hline Age at diabetes onset (years) & $18.4 \pm 9.4(1.0-35.0)$ & NA \\
\hline $\mathrm{HbA}_{1 \mathrm{c}}(\mathrm{mmol} / \mathrm{mol})$ & $64.5 \pm 15.7(37.0-95.0)^{* * *}$ & $32.9 \pm 2.8(28.0-39.0)$ \\
\hline $\mathrm{HbA}_{1 \mathrm{c}}(\%)$ & $8.0 \pm 1.4(5.5-10.8)^{* * *}$ & $5.2 \pm 0.3(4.7-5.7)$ \\
\hline Insulin delivery (CSII/MDI) & $9 / 13$ & NA \\
\hline Total insulin dose $\left(\mathrm{U} \mathrm{kg}^{-1} \mathrm{day}^{-1}\right)$ & $0.67 \pm 0.19(0.24-0.96)$ & NA \\
\hline Long-acting insulin analogue (MDI) or insulin basal rate (CSII) $\left(\mathrm{U} \mathrm{kg}^{-1}\right.$ day $\left.^{-1}\right)$ & $0.28 \pm 0.13(0.11-0.64)$ & NA \\
\hline Rapid-acting insulin analogue (MDI) or insulin bolus (CSII) $\left(\mathrm{U} \mathrm{kg}^{-1}\right.$ day $\left.^{-1}\right)$ & $0.39 \pm 0.15(0.00-0.65)$ & NA \\
\hline \multicolumn{3}{|l|}{ Fasting lipid profile in serum } \\
\hline Triacylglycerol (mmol/l) & $0.96 \pm 0.40(0.49-2.10)$ & $0.99 \pm 0.25(0.54-1.44)$ \\
\hline Cholesterol $(\mathrm{mmol} / \mathrm{l})$ & $4.61 \pm 0.73(3.44-6.07)$ & $4.55 \pm 0.60(3.57-5.71)$ \\
\hline $\mathrm{HDL}-\mathrm{C}(\mathrm{mmol} / \mathrm{l})$ & $1.43 \pm 0.31(0.98-2.09)$ & $1.35 \pm 0.31(0.88-2.15)$ \\
\hline LDL-C (mmol/l) & $2.74 \pm 0.62(1.78-4.16)$ & $2.75 \pm 0.54(1.89-3.83)$ \\
\hline ApoA-1 (mmol/1) & $1.61 \pm 0.21(1.30-2.20)^{*}$ & $1.48 \pm 0.21(1.15-1.89)$ \\
\hline ApoB $(\mathrm{mmol} / \mathrm{l})$ & $0.74 \pm 0.15(0.42-0.97)$ & $0.73 \pm 0.14(0.46-0.98)$ \\
\hline Lipoprotein(a) (mg/l) & $194.87 \pm 224.70(93.1-1030.00)$ & $185.21 \pm 160.10(93.1-576.00)$ \\
\hline \multicolumn{3}{|l|}{ Physical activity and aerobic fitness } \\
\hline$V \mathrm{O}_{2 \max }\left(\mathrm{ml} \mathrm{min}^{-1} \mathrm{~kg}^{-1}\right)$ & $35.6 \pm 7.7(25.0-52.0) * *$ & $39.6 \pm 6.8(31.0-49.0)$ \\
\hline Exercise duration (min) & $19.1 \pm 4.3(11.5-26.0)$ & $21.5 \pm 4.6(14.0-31.0)$ \\
\hline Total activity by MAQ (MET h/week) & $43.0 \pm 87.0(1.2-384.0)$ & $55.3 \pm 52.3(0.0-176.0)$ \\
\hline MVPA by accelerometery ( $\mathrm{min} / \mathrm{week})$ & $234.9 \pm 160.0(35.0-623.0)$ & $280.1 \pm 114.9(131.0-558.0)$ \\
\hline Sedentary time by accelerometery (h/day) & $9.1 \pm 2.3(7.0-15.1)$ & $10.4 \pm 2.2(7.2-14.2)$ \\
\hline \multicolumn{3}{|l|}{ Usual daily nutrient intake } \\
\hline Total caloric $(\mathrm{TC})$ intake $(\mathrm{kJ})$ & $8718.7 \pm 2183.5$ & $9061.8 \pm 1890.1$ \\
\hline Protein $(\%$ of TC) & $16.2 \pm 3.5$ & $16.3 \pm 3.3$ \\
\hline Protein $(g / k g)$ & $1.07 \pm 0.25$ & $1.19 \pm 0.34$ \\
\hline Fat $(\%$ of TC) & $37.1 \pm 5.7$ & $38.3 \pm 4.5$ \\
\hline Polyunsaturated/saturated fatty acid ratio & $0.3 \pm 0.1$ & $0.4 \pm 0.1$ \\
\hline Cholesterol (mg) & $323.1 \pm 137.1$ & $320.9 \pm 145.3$ \\
\hline Carbohydrate (\% of TC) & $47.7 \pm 6.2$ & $46.7 \pm 5.7$ \\
\hline High glycaemic index carbohydrate (\% of TC) & $16.2 \pm 5.3$ & $18.1 \pm 4.7$ \\
\hline \multirow{2}{*}{\multicolumn{3}{|c|}{ Vitamins }} \\
\hline & & \\
\hline Vitamin A $(\mu \mathrm{g})$ & $304.5 \pm 95.4$ & $367.2 \pm 195.7$ \\
\hline Vitamin $B_{1}(\mathrm{mg})$ & $1.3 \pm 0.5$ & $1.3 \pm 0.4$ \\
\hline Vitamin $B_{2}(\mathrm{mg})$ & $1.7 \pm 0.4$ & $1.8 \pm 0.5$ \\
\hline Vitamin $B_{3}(\mathrm{mg})$ & $18.2 \pm 7.3$ & $18.2 \pm 6.9$ \\
\hline Vitamin $\mathrm{B}_{5}(\mathrm{mg})$ & $5.5 \pm 1.1$ & $5.7 \pm 1.5$ \\
\hline Vitamin $B_{6}(\mathrm{mg})$ & $2.0 \pm 0.6$ & $1.8 \pm 0.5$ \\
\hline Vitamin $B_{9}(\mu g)$ & $293.4 \pm 85.6$ & $303.6 \pm 78.0$ \\
\hline Vitamin $B_{12}(\mu \mathrm{g})$ & $4.3 \pm 1.6$ & $5.8 \pm 4.2$ \\
\hline Vitamin C (mg) & $66.1 \pm 34.6$ & $82.3 \pm 51.5$ \\
\hline Vitamin D ( $\mu \mathrm{g})$ & $10.9 \pm 2.0$ & $10.8 \pm 1.6$ \\
\hline Vitamin E (mg) & $12.6 \pm 6.4$ & $11.9 \pm 4.3$ \\
\hline \multicolumn{3}{|l|}{ Minerals } \\
\hline Magnesium (mg) & $271.9 \pm 68.7$ & $283.5 \pm 56.0$ \\
\hline Potassium (mg) & $2661.7 \pm 636.0$ & $2768.3 \pm 641.2$ \\
\hline Sodium (mg) & $5015.0 \pm 798.0$ & $5182.4 \pm 795.6$ \\
\hline Selenium $(\mu \mathrm{g})$ & $135.2 \pm 91.9$ & $149.2 \pm 57.1$ \\
\hline \multicolumn{3}{|l|}{ Others } \\
\hline Polyphenols (mg) & $896.5 \pm 656.9$ & $890.7 \pm 782.9$ \\
\hline Flavonoids (mg) & $197.3 \pm 257.8$ & $360.9 \pm 504.8$ \\
\hline Nitrite $(\mathrm{mg})$ & $1.6 \pm 0.9$ & $1.5 \pm 0.8$ \\
\hline Nitrate (mg) & $67.3 \pm 54.6$ & $47.0 \pm 42.2$ \\
\hline
\end{tabular}

Data are mean \pm SD or number of participants for some outcomes; (min-max) is also added for some outcomes

Values significantly different from those of the healthy individuals using Student's unpaired $t$ tests or Mann-Whitney $U$ tests: $* p<0.05 ; * *<0.01$; $* * * p<0.001$

Physical activity level: Accelerometery data are displayed only for 14 patients with type 1 diabetes and 16 healthy individuals because some participants did not strictly follow our recommendations (mainly, wearing the accelerometer during all waking hours) and some accelerometer devices were malfunctional (no signal was recorded at the end of the week)

ApoA-1, Apolipoprotein A1; ApoB, Apolipoprotein B; CSII, continuous subcutaneous insulin infusion; MAQ, Modified Activity Questionnaire, MDI, multiple daily insulin injection; MET, metabolic equivalent of task; MVPA, moderate to vigorous physical activity; NA, not applicable 
was collected for determination of the 'so-called' arterial markers.

Statistical analyses Statistical analyses were performed using SPSS software (version 19). Results are reported as mean \pm SD. Normality was checked with Shapiro-Wilk test. Fasting data were compared between groups using Student's unpaired $t$ tests or Mann-Whitney $U$ tests according to data distribution. A logarithmic transformation $\left(\log _{10}\right)$ was applied if the distribution of the residuals was not Gaussian. Using linear mixed models, postprandial data were compared between groups (fixed effect) and in response to exercise as a fixed effect (for $\Delta \mathrm{THb}$ and $\Delta \mathrm{HHb}$, according to exercise intensity with a value for every $10 \% \dot{V} \mathrm{O}_{2 \max }$, as well as according to absolute exercise power [Watts]; for blood outcomes, with categories rest, peak exercise and, when available, recovery). A random intercept was included for each individual to consider the repetition of the measures performed on the same participant. The interaction between group and exercise was also included as a fixed effect to allow the effect of exercise to vary between groups. Within each group, to study the involvement of sex, aerobic fitness $\left(\dot{V} \mathrm{O}_{2 \max }\right)$, and, for people with diabetes, $\mathrm{HbA}_{1 \mathrm{c}}$, we successively added each of these variables (sequentially) as covariates in a mixed model with exercise and interaction between each variable and exercise. If significant main effects or interactions were observed, Bonferroni post hoc comparisons were applied. Pairwise correlations were tested using Pearson's $r$ or, for nonparametric data, Spearman's $\rho$. $p<0.05$ was considered statistically significant.

\section{Results}

Participants' characteristics Anthropometric data (including body composition), demographic data, smoking status and diet did not differ between groups (Table 1). Despite comparable levels of habitual physical activity (accelerometery), participants with type 1 diabetes displayed lower $\dot{V} \mathrm{O}_{2 \max }$ than healthy individuals did (Table 1).

Exercise-induced muscle vasoreactivity Muscle blood volume increased throughout exercise, from $70 \%$ and from $50 \%$ of $\dot{V} \mathrm{O}_{2 \max }$ in participants with and without diabetes, respectively, but this increase was lower among those with diabetes (main effect 'exercise', $p<0.001$, interaction 'exercise' $\times$ 'group', $p<0.05$, when NIRS was studied according to relative exercise intensities, with comparable results for absolute intensities; e.g. at peak exercise, $\Delta \mathrm{THb} 2.03 \pm 5.82$ in diabetic participants vs $5.33 \pm 5.54 \mu \mathrm{mol} / 1$ in control participants).

Markers of NO- bioavailability and factors influencing NO. metabolism or vasoreactivity The impaired exercise-induced muscle vasoreactivity in type 1 diabetes was accompanied by lower arterial $\mathrm{K}^{+}$and lower plasma L-arginine levels at postprandial rest and after maximal exercise (Table 2, Fig. 1). In addition to L-arginine, its derivatives, ADMA, SDMA and Lcitrulline were also reduced in type 1 diabetes (Fig. 1). Lhomoarginine was also measured, with no significant intergroup difference in the fasting state, and for postprandial values, a significant main effect for exercise $(p<0.001)$, no significant effect of group $(p=0.061)$ and no significant interaction (values in the group with type 1 diabetes, fasting rest $1.4 \pm 0.4$, postprandial rest $1.4 \pm 0.4$, peak exercise $1.2 \pm 0.4$; recovery $1.2 \pm 0.3 \mu \mathrm{mol} / \mathrm{l}$; values in the healthy individuals, fasting rest $1.6 \pm 0.6$; postprandial rest $1.6 \pm 0.5$, peak exercise $1.4 \pm 0.5$, recovery $1.4 \pm 0.4 \mu \mathrm{mol} / \mathrm{l})$.

Serum RXNO were decreased in the fasting state in diabetic vs non-diabetic participants (Table 2). However, RXNO, nitrite and nitrate did not differ between groups in the postprandial state in response to exercise and recovery (Table 2). Of note, the $\triangle \mathrm{HHb}$ increase was blunted in diabetic vs heathy individuals (main effect 'exercise', $p<0.001$, interaction 'exercise' $\times$ 'group', $p<0.001$, whatever NIRS was studied according to absolute or relative exercise intensities), despite a lower $\mathrm{PaO}_{2}$ (Table 2). MetHb (\%) did not differ according to group or exercise (all main effects NS, data not shown). Arterial $\mathrm{HCO}_{3}{ }^{-}$ decreased with exercise $(p<0.001)$ without any intergroup difference (data not shown). While nitrite and RXNO concentrations did not change in response to exercise, serum nitrate decreased in response to exercise in both groups (Table 2).

Oxidative stress biomarkers As free radicals and oxidative stress may decrease $\mathrm{NO} \bullet$ availability, we studied a wealth of antioxidant readouts and oxidative stress markers. In both groups, exercise was associated with a decrease in some of the non-enzymatic antioxidants and an increase in erythrocyte glutathione reductase activity (Table 2). Oxidative stress biomarkers did not increase in response to exercise in either group, while serum CML levels even decreased (Table 2). Erythrocyte glutathione reductase activity was higher in cases of higher aerobic fitness among the participants with type 1 diabetes (covariate $\dot{V} \mathrm{O}_{2 \max }$, mean estimation: $+0.19, p<0.001$ ). While postprandial (at rest, after exercise and during recovery) levels of oxidative stress biomarkers did not differ between groups (Table 2), plasma urate concentrations (and hence plasma total antioxidant status) was lower in diabetic vs non-diabetic individuals (Fig. 2). In the fasting resting state, patients' plasma urate was reduced (Fig. 2) and this was also the case for serum vitamin $\mathrm{E}$ corrected by cholesterol and triacylglycerols, while plasma 8 -iso-PGF2 $\alpha$ was higher in diabetic participants than in healthy participants (Table 2). The fasting lipid profile was comparable between groups (Table 1), except for the antiatherogenic apolipoprotein A1 (Table 1) and the lipolysis marker, glycerol (Table 2), which were higher in participants with diabetes. 
Table 2 Bioavailability of $\mathrm{NO} \bullet$ and factors influencing $\mathrm{NO} \bullet$ metabolism or vasoreactivity at rest (fasting and postprandial) and in response to incremental maximal exercise (postprandial)

\begin{tabular}{|c|c|c|c|c|}
\hline & & Participants with type 1 diabetes & Healthy participants & $p$ values \\
\hline \multicolumn{5}{|l|}{ Bioavailability of $\mathrm{NO} \bullet$} \\
\hline \multirow{4}{*}{ Serum nitrite $(\mu \mathrm{mol} / \mathrm{l})$} & Fasting rest & $0.24 \pm 0.19$ & $0.18 \pm 0.09$ & NS \\
\hline & Postprandial rest $\mathrm{t}^{\mathrm{a}}$ & $0.19 \pm 0.12$ & $0.18 \pm 0.10$ & Exercise: NS \\
\hline & Postprandial peak exercise $^{\mathrm{a}}$ & $0.16 \pm 0.11$ & $0.14 \pm 0.06$ & Group: NS \\
\hline & Postprandial recovery ${ }^{\mathrm{a}}$ & $0.18 \pm 0.11$ & $0.17 \pm 0.11$ & Interaction: NS \\
\hline \multirow[t]{4}{*}{ Serum nitrate $(\mu \mathrm{mol} / 1)^{\mathrm{b}}$} & Fasting rest & $39.3 \pm 11.7$ & $37.1 \pm 8.8$ & NS \\
\hline & Postprandial rest ${ }^{\mathrm{a}}$ & $39.7 \pm 11.7$ & $34.1 \pm 11.8$ & Exercise $<0.01$ \\
\hline & Postprandial peak exercise $^{\mathrm{a}}$ & $32.3 \pm 10.5$ & $31.6 \pm 14.0$ & Group: NS \\
\hline & Postprandial recovery ${ }^{\mathrm{a}}$ & $33.8 \pm 10.5$ & $34.7 \pm 15.1$ & Interaction: NS \\
\hline \multirow[t]{4}{*}{ Serum RXNO (nmol/l) } & Fasting rest & $11.2 \pm 4.6$ & $15.2 \pm 6.6$ & $<0.05$ (group) \\
\hline & Postprandial rest ${ }^{\mathrm{a}}$ & $12.1 \pm 4.4$ & $12.7 \pm 6.5$ & Exercise: NS \\
\hline & Postprandial peak exercise $^{\mathrm{a}}$ & $12.6 \pm 4.9$ & $12.2 \pm 5.0$ & Group: NS \\
\hline & Postprandial recovery ${ }^{\mathrm{a}}$ & $11.7 \pm 3.7$ & $10.8 \pm 3.1$ & Interaction: NS \\
\hline \multirow[t]{4}{*}{$\mathrm{L}$-arginine/ADMA ratio } & Fasting rest & $163.7 \pm 39.8$ & $157.5 \pm 22.5$ & NS \\
\hline & Postprandial rest & $129.5 \pm 32.0$ & $141.6 \pm 27.8$ & Exercise $p<0.001$ \\
\hline & Postprandial peak exercise & $99.8 \pm 27.3$ & $105.5 \pm 29.0$ & Group: NS \\
\hline & Postprandial recovery & $107.5 \pm 26.0$ & $124.3 \pm 24.9$ & Interaction: NS \\
\hline \multicolumn{5}{|l|}{ Oxidative stress biomarkers } \\
\hline \multirow[t]{4}{*}{ Plasma MDA $(\mu \mathrm{mol} / 1)$} & Fasting rest ${ }^{\mathrm{a}}$ & $0.56 \pm 0.15$ & $0.58 \pm 0.13$ & NS \\
\hline & Postprandial rest ${ }^{\mathrm{a}}$ & $0.61 \pm 0.21$ & $0.66 \pm 0.20$ & Exercise: NS \\
\hline & Postprandial peak exercise $^{\mathrm{a}}$ & $0.58 \pm 0.13$ & $0.70 \pm 0.27$ & Group: NS \\
\hline & Postprandial recovery ${ }^{\mathrm{a}}$ & $0.63 \pm 0.36$ & $0.66 \pm 0.21$ & Interaction: NS \\
\hline \multirow[t]{4}{*}{ Plasma free 8 -iso-PGF2 $\alpha(\mathrm{pg} / \mathrm{ml})$} & Fasting rest & $10.45 \pm 8.13$ & $4.90 \pm 5.16$ & $<0.05$ (group) \\
\hline & Postprandial rest & $4.79 \pm 3.47$ & $5.46 \pm 4.12$ & Exercise: NS \\
\hline & Postprandial peak exercise & $5.18 \pm 4.90$ & $8.08 \pm 8.95$ & Group: NS \\
\hline & Postprandial recovery & $5.85 \pm 4.52$ & $7.49 \pm 6.18$ & Interaction: NS \\
\hline \multirow{4}{*}{$\begin{array}{l}\text { Plasma fluorescent oxidation } \\
\text { products }(\mathrm{FI} / \mathrm{ml})\end{array}$} & Fasting rest & $130.7 \pm 41.2$ & $150.0 \pm 70.9$ & NS \\
\hline & Postprandial rest & $127.0 \pm 47.5$ & $130.3 \pm 37.2$ & Exercise: NS \\
\hline & Postprandial peak exercise & $126.7 \pm 53.2$ & $122.4 \pm 37.8$ & Group: NS \\
\hline & Postprandial recovery & $135.1 \pm 58.8$ & $128.3 \pm 33.4$ & Interaction: NS \\
\hline \multirow[t]{4}{*}{ Serum CML (pmol/ml) } & Fasting rest $\mathrm{t}^{\mathrm{a}}$ & $51.7 \pm 44.2$ & $64.7 \pm 67.2$ & NS \\
\hline & Postprandial rest ${ }^{\mathrm{a}}$ & $71.0 \pm 53.7$ & $71.8 \pm 61.9$ & Exercise $:<0.01$ \\
\hline & Postprandial peak exercise $^{a}$ & $54.9 \pm 47.2$ & $60.2 \pm 48.5$ & Group: NS \\
\hline & Postprandial recovery ${ }^{\mathrm{a}}$ & $52.7 \pm 48.9$ & $56.9 \pm 51.3$ & Interaction: NS \\
\hline \multicolumn{5}{|l|}{ Antioxidant variables ${ }^{c}$} \\
\hline \multirow[t]{4}{*}{ Erythrocyte SOD (U/g Hb) } & Fasting rest ${ }^{\mathrm{a}}$ & $1808.5 \pm 194.6$ & $2022.3 \pm 485.1$ & NS \\
\hline & Postprandial rest ${ }^{\mathrm{a}}$ & $1832.9 \pm 233.2$ & $1963.5 \pm 388.0$ & Exercise: NS \\
\hline & Postprandial peak exercise $^{\mathrm{a}}$ & $1711.4 \pm 292.6$ & $1870.9 \pm 195.7$ & Group: NS \\
\hline & Postprandial recovery ${ }^{\mathrm{a}}$ & $1785.6 \pm 349.0$ & $1864.3 \pm 280.7$ & Interaction: NS \\
\hline \multirow[t]{4}{*}{ Erythrocyte GPx (U/g Hb) } & Fasting rest ${ }^{\mathrm{a}}$ & $61.13 \pm 11.05$ & $59.88 \pm 11.04$ & NS \\
\hline & Postprandial rest ${ }^{\mathrm{a}}$ & $58.88 \pm 13.55$ & $62.67 \pm 10.53$ & Exercise: NS \\
\hline & Postprandial peak exercise $^{\mathrm{a}}$ & $59.50 \pm 9.28^{\dagger \dagger}$ & $60.13 \pm 8.02^{\dagger \dagger}$ & Group: NS \\
\hline & Postprandial recovery ${ }^{\mathrm{a}}$ & $61.75 \pm 10.27^{*}$ & $58.00 \pm 8.39^{\dagger \dagger \dagger}$ & Interaction: $<0.05$ \\
\hline \multirow[t]{4}{*}{ Erythrocyte GR (U/g Hb) } & Fasting rest $\mathrm{s}^{\mathrm{a}}$ & $5.83 \pm 1.28$ & $5.74 \pm 0.98$ & NS \\
\hline & Postprandial rest ${ }^{\mathrm{a}}$ & $5.47 \pm 1.35$ & $5.71 \pm 0.89$ & Exercise $<0.01$ \\
\hline & Postprandial peak exercise $^{\mathrm{a}}$ & $5.74 \pm 1.28$ & $5.92 \pm 0.87$ & Group: NS \\
\hline & Postprandial recovery ${ }^{\mathrm{a}}$ & $5.58 \pm 1.22$ & $5.42 \pm 1.47$ & Interaction: NS \\
\hline \multirow[t]{4}{*}{ Plasma vitamin $C(\mu \mathrm{mol} / \mathrm{l})$} & Fasting rest $^{\mathrm{a}}$ & $48.5 \pm 13.3$ & $50.4 \pm 15.9$ & NS \\
\hline & Postprandial rest ${ }^{\mathrm{a}}$ & $57.5 \pm 26.2$ & $56.6 \pm 21.9$ & Exercise $:<0.01$ \\
\hline & Postprandial peak exercise ${ }^{a}$ & $55.3 \pm 15.7$ & $54.0 \pm 22.1$ & Group: NS \\
\hline & Postprandial recovery ${ }^{\mathrm{a}}$ & $61.4 \pm 18.0$ & $61.4 \pm 22.1$ & Interaction: NS \\
\hline Serum vitamin $E(\mu \mathrm{mol} / \mathrm{l})$ & Fasting rest & $26.87 \pm 4.08$ & $24.27 \pm 3.59$ & $<0.05$ (group) \\
\hline & Postprandial rest ${ }^{\mathrm{a}}$ & $27.05 \pm 5.19$ & $24.73 \pm 3.45$ & Exercise $:<0.001$ \\
\hline & Postprandial peak exercise $^{\mathrm{a}}$ & $25.19 \pm 4.76$ & $22.73 \pm 3.06$ & Group: NS \\
\hline & Postprandial recovery ${ }^{\mathrm{a}}$ & $26.70 \pm 3.86$ & $24.00 \pm 2.03$ & Interaction: NS \\
\hline $\begin{array}{l}\text { Vitamin } \mathrm{E}(\mu \mathrm{mol} / \mathrm{l}) /(\mathrm{cholesterol}+ \\
\text { triacylglycerol })(\mathrm{mmol} / \mathrm{l}) \text { ratio }\end{array}$ & Fasting rest & $4.91 \pm 0.71$ & $5.39 \pm 0.55$ & $<0.05$ (group) \\
\hline Serum vitamin $A(\mu \mathrm{mol} / 1)$ & Fasting rest $^{\mathrm{a}}$ & $1.64 \pm 0.41$ & $2.12 \pm 0.36$ & $<0.01$ (group) \\
\hline & Postprandial rest ${ }^{\mathrm{a}}$ & $1.71 \pm 0.36$ & $2.14 \pm 0.34$ & Exercise $:<0.01$ \\
\hline & Postprandial peak exercise $^{\mathrm{a}}$ & $1.60 \pm 0.41$ & $2.04 \pm 0.29$ & Group: $<0.01$ \\
\hline & Postprandial recovery ${ }^{\mathrm{a}}$ & $1.70 \pm 0.40$ & $2.13 \pm 0.36$ & Interaction: NS \\
\hline Serum T-SH $(\mu \mathrm{mol} / \mathrm{l})$ & Fasting rest & $356.00 \pm 35.10$ & $373.40 \pm 36.15$ & NS \\
\hline & Postprandial rest ${ }^{\mathrm{a}}$ & $353.64 \pm 43.12$ & $377.60 \pm 44.16$ & Exercise $:<0.01$ \\
\hline & Postprandial peak exercise ${ }^{a}$ & $319.84 \pm 42.68$ & $344.55 \pm 63.07$ & Group: NS \\
\hline
\end{tabular}


Table 2 (continued)

\begin{tabular}{|c|c|c|c|c|}
\hline & & Participants with type 1 diabetes & Healthy participants & $p$ values \\
\hline \multirow{4}{*}{$\begin{array}{l}\mathrm{T}-\mathrm{SH} / \text { protein ratio }\left(\mu \mathrm{mol} \mathrm{l}{ }^{-1} \mathrm{~g}\right. \\
\left.\text { protein }^{-1}\right)^{\mathrm{d}}\end{array}$} & Postprandial recovery ${ }^{\mathrm{a}}$ & $341.77 \pm 55.33$ & $365.91 \pm 55.61$ & Interaction: NS \\
\hline & Fasting rest & $4.92 \pm 0.55$ & $5.01 \pm 0.47$ & NS \\
\hline & Postprandial rest ${ }^{\mathrm{a}}$ & $4.76 \pm 0.64$ & $4.91 \pm 0.67$ & Exercise: $<0.01$ \\
\hline & Postprandial peak exercise ${ }^{a}$ & $4.11 \pm 0.62$ & $4.30 \pm 0.88$ & Group: NS \\
\hline \multirow{5}{*}{$\begin{array}{l}\text { Total antioxidant status and } \\
\text { urate subtraction }(\mathrm{mmol} / \mathrm{l})^{\mathrm{c}, \mathrm{e}}\end{array}$} & Postprandial recovery ${ }^{\mathrm{a}}$ & $3.86 \pm 1.53$ & $4.41 \pm 1.49$ & Interaction: NS \\
\hline & Fasting rest & $1.34 \pm 0.29$ & $1.39 \pm 0.36$ & NS \\
\hline & Postprandial rest & $1.36 \pm 0.33$ & $1.50 \pm 0.39$ & Exercise $:<0.01$ \\
\hline & Postprandial peak exercise & $1.23 \pm 0.29^{\dagger \dagger}$ & $1.34 \pm 0.35^{\dagger \dagger}$ & Group: NS \\
\hline & Postprandial recovery & $1.36 \pm 0.32$ & $1.39 \pm 0.39^{\dagger \dagger}$ & Interaction: $<0.05$ \\
\hline \multicolumn{5}{|l|}{$\begin{array}{l}\text { Other substances with vasoactive } \\
\text { properties }\end{array}$} \\
\hline \multirow[t]{2}{*}{ Plasma adrenaline $(\mathrm{pmol} / \mathrm{l})^{\mathrm{b}}$} & Postprandial rest & $709.0 \pm 709.0$ & $545.4 \pm 490.9$ & $\begin{array}{l}\text { Exercise: }<0.001 \\
\text { Group: NS }\end{array}$ \\
\hline & Postprandial peak exercise & $1199.9 \pm 818.1$ & $1199.9 \pm 818.1$ & Interaction: NS \\
\hline Plasma noradrenaline (pmol/l) & Postprandial rest & $2247.3 \pm 1123.7$ & $2957.0 \pm 2247.3$ & $\begin{array}{l}\text { Exercise: }<0.001 \\
\text { Group: NS }\end{array}$ \\
\hline & Postprandial peak exercise & $8752.7 \pm 4731.2$ & $9344.1 \pm 4317.2$ & Interaction: NS \\
\hline \multirow[t]{3}{*}{ Serum adiponectin $(\mu \mathrm{g} / \mathrm{ml})$} & Fasting rest & $10.8 \pm 5.8$ & $8.2 \pm 4.4$ & NS \\
\hline & Postprandial rest & $10.6 \pm 5.6$ & $8.8 \pm 4.7$ & Exercise $:<0.05$ \\
\hline & Postprandial peak exercise & $9.9 \pm 4.5$ & $8.3 \pm 4.3$ & Group: NS \\
\hline & Postprandial recovery & $10.5 \pm 4.6$ & $8.0 \pm 3.2$ & Interaction: NS \\
\hline \multirow[t]{4}{*}{ Serum leptin (ng/ml) } & Fasting rest & $7.2 \pm 6.9$ & $5.9 \pm 6.3$ & NS \\
\hline & Postprandial rest & $7.3 \pm 6.0$ & $4.9 \pm 3.9$ & Exercise: $<0.001$ \\
\hline & Postprandial peak exercise & $6.8 \pm 5.4$ & $4.1 \pm 3.0$ & Group: NS \\
\hline & Postprandial recovery & $6.4 \pm 5.4$ & $3.3 \pm 2.5$ & Interaction: NS \\
\hline \multirow[t]{4}{*}{ Serum free insulin $(\mathrm{pmol} / \mathrm{l})$} & Fasting rest & $235.5 \pm 363.1$ & $50.5 \pm 19.0$ & $<0.05$ (group) \\
\hline & Postprandial rest & $253.3 \pm 293.6$ & $72.6 \pm 37.1$ & Exercise: NS \\
\hline & Postprandial peak exercise & $250.6 \pm 308.4$ & $69.2 \pm 47.9$ & Group: $<0.01$ \\
\hline & Postprandial recovery & $254.3 \pm 282.7$ & $77.1 \pm 43.5$ & Interaction: NS \\
\hline \multirow[t]{2}{*}{ Arterial $\mathrm{pH}$} & Postprandial rest & $7.41 \pm 0.04$ & $7.42 \pm 0.02$ & $\begin{array}{l}\text { Exercise: }<0.001 \\
\text { Group: NS }\end{array}$ \\
\hline & Postprandial peak exercise & $7.26 \pm 0.04$ & $7.28 \pm 0.07$ & Interaction: NS \\
\hline \multirow[t]{2}{*}{$\mathrm{PaO}_{2}(\mathrm{mmHg})^{\mathrm{b}}$} & Postprandial rest & $94.7 \pm 10.9$ & $99.9 \pm 9.2$ & $\begin{array}{l}\text { Exercise }:<0.001 \\
\text { Group }<0.05\end{array}$ \\
\hline & Postprandial peak exercise & $103.8 \pm 13.6^{\dagger}$ & $109.2 \pm 10.9^{\dagger \dagger}$ & Interaction: NS \\
\hline \multirow[t]{2}{*}{$\mathrm{PaCO}_{2}(\mathrm{mmHg})^{\mathrm{b}}$} & Postprandial rest & $38.3 \pm 2.1$ & $36.9 \pm 4.5$ & $\begin{array}{l}\text { Exercise: }<0.001 \\
\text { Group: NS }\end{array}$ \\
\hline & Postprandial peak exercise & $31.1 \pm 4.5$ & $29.7 \pm 4.1$ & Interaction: NS \\
\hline \multirow[t]{2}{*}{ Arterial $\mathrm{K}^{+}(\mathrm{mmol} / \mathrm{l})$} & Postprandial rest & $5.0 \pm 0.5$ & $5.9 \pm 1.0$ & $\begin{array}{l}\text { Exercise }:<0.001 \\
\text { Group: }<0.05\end{array}$ \\
\hline & Postprandial peak exercise & $4.6 \pm 0.5^{\dagger \dagger}$ & $5.4 \pm 0.8^{\dagger}$ & Interaction: NS \\
\hline \multirow[t]{2}{*}{ Arterial lactate $(\mathrm{mmol} / \mathrm{l})$} & Postprandial rest & $1.3 \pm 0.4$ & $0.9 \pm 0.3$ & $\begin{array}{l}\text { Exercise: }<0.001 \\
\text { Group: NS }\end{array}$ \\
\hline & Postprandial peak exercise & $11.8 \pm 3.0$ & $11.3 \pm 3.3$ & Interaction: NS \\
\hline \multicolumn{5}{|l|}{ Metabolic data } \\
\hline \multirow[t]{4}{*}{ Plasma glucose (mmol/l) } & Fasting rest & $6.5 \pm 3.4$ & $5.0 \pm 0.7$ & NS \\
\hline & Postprandial rest & $7.0 \pm 2.7$ & $5.0 \pm 0.6$ & Exercise: NS \\
\hline & Postprandial peak exercise & $7.1 \pm 1.8$ & $5.3 \pm 0.8$ & Group: $<0.001$ \\
\hline & Postprandial recovery & $7.3 \pm 2.0$ & $5.5 \pm 1.3$ & Interaction: NS \\
\hline \multirow[t]{4}{*}{ Serum NEFA (mmol/l) } & Fasting rest & $0.60 \pm 0.33$ & $0.41 \pm 0.15$ & NS \\
\hline & Postprandial rest & $0.29 \pm 0.19$ & $0.35 \pm 0.18$ & Exercise: $<0.001$ \\
\hline & Postprandial peak exercise & $0.18 \pm 0.08$ & $0.31 \pm 0.14$ & Group: NS \\
\hline & Postprandial recovery & $0.32 \pm 0.27$ & $0.38 \pm 0.17$ & Interaction: NS \\
\hline \multirow[t]{3}{*}{ Serum glycerol (mg/l) ${ }^{\mathrm{b}}$} & Fasting rest & $5.57 \pm 3.45$ & $3.05 \pm 1.92$ & $<0.05$ (group) \\
\hline & Postprandial rest & $3.06 \pm 1.96$ & $2.64 \pm 1.54$ & Exercise $:<0.001$ \\
\hline & Postprandial peak exercise & $4.77 \pm 2.29$ & $6.98 \pm 3.10^{\dagger \dagger}$ & Group: NS \\
\hline \multirow{5}{*}{ Plasma glucagon (ng/l) ${ }^{\mathrm{b}}$} & Postprandial recovery & $8.20 \pm 3.34^{\dagger \dagger}+4$ & $8.84 \pm 3.13^{\dagger \dagger}$ & Interaction $<0.05$ \\
\hline & Fasting rest & $264.7 \pm 113.7$ & $181.7 \pm 85.7$ & $<0.05$ (group) \\
\hline & Postprandial rest & $211.0 \pm 80.5$ & $209.9 \pm 108.2$ & Exercise $:<0.001$ \\
\hline & Postprandial peak exercise & $180.2 \pm 62.9$ & $217.4 \pm 98.5$ & Group: NS \\
\hline & Postprandial recovery & $228.8 \pm 107.9$ & $347.5 \pm 243.8$ & Interaction: NS \\
\hline $\begin{array}{l}\text { Data for calculating exercise-induc } \\
\text { change in serum and plasma vo }\end{array}$ & & & & \\
\hline Venous $\mathrm{Hb}(\mathrm{g} / \mathrm{l})$ & Postprandial rest & $148.7 \pm 13.2$ & $146.4 \pm 11.5$ & Exercise: $<0.001$ \\
\hline & Postprandial peak exercise & $161.2 \pm 14.4$ & $159.6 \pm 12.4$ & Group: NS \\
\hline
\end{tabular}


Table 2 (continued)

\begin{tabular}{llccc}
\hline & & Participants with type 1 diabetes & Healthy participants & $p$ values \\
\hline & Postprandial recovery & $151.3 \pm 14.2$ & $150.8 \pm 14.0$ & Interaction: NS \\
Venous haematocrit (\%) & Postprandial rest & $45.3 \pm 4.1$ & $44.9 \pm 3.4$ & Exercise: $<0.001$ \\
& Postprandial peak exercise & $48.9 \pm 4.5$ & $49.1 \pm 3.5$ & Group: NS \\
& Postprandial recovery & $46.5 \pm 4.5$ & $46.2 \pm 4.2$ & Interaction: NS \\
\hline
\end{tabular}

Data are mean $\pm \mathrm{SD}$

$p$ values in the right-hand column are mixed-model main effects for postprandial values or intergroup difference with Student's unpaired $t$ tests/MannWhitney $U$ tests for fasting values

Main effects from mixed models (for postprandial values) include $p$ values for an exercise effect (Exercise), a group effect (Group), and an exercise $\times$ group interaction (Interaction)

Pairwise post hoc analyses when an interaction or both exercise and group effects were found: time effect, significantly different from those at rest at ${ }^{\dagger} p<0.05 ;{ }^{\dagger \dagger} p<0.01$, or ${ }^{\dagger \dagger \dagger} p<0.001$; significantly different from those at peak exercise at ${ }^{\star} p<0.05 ;{ }^{\star \dagger} p<0.01$, or ${ }^{\star+\ddagger} p<0.001$

Post hoc analyses, which were performed if an exercise effect was exclusively found, are detailed in ESM Table 3

${ }^{a}$ The number of participants for whom the assay was performed is lower than the total sample size, because of limited quantity of blood sampled. Details of the exact numbers of participants for these outcomes are provided in ESM Table 4

${ }^{\mathrm{b}}$ A logarithmic transformation was applied to data with a non-Gaussian distribution

${ }^{\mathrm{c}}$ The specific values of total antioxidant status and urate are separately displayed in Fig. 2

${ }^{\mathrm{d}}$ Normalised to plasma protein

${ }^{\mathrm{e}}$ Corrected by urate

'Fasting rest' means during the second visit, in a fasting resting state; 'Postprandial rest', 'Postprandial peak exercise' and 'Postprandial recovery' mean during the first visit, in a postprandial state, at rest before the exercise, at peak of exercise and after 15 min of passive recovery, respectively

$\mathrm{FI} / \mathrm{ml}$, relative fluorescent intensity per ml plasma; GPx, glutathione peroxidase; GR, glutathione reductase; MDA, malondialdehyde, equivalent to malondialdehyde-thiobarbituric adducts; T-SH: total free thiols

Influences of $\mathrm{HbA}_{1 \mathrm{c}}$ and sex In individuals with type 1 diabetes, a higher $\mathrm{HbA}_{1 \mathrm{c}}$ value was a negative determinant of postprandial concentrations of L-arginine (mean estimation: -4.0 , $p<0.05)$. $\mathrm{HbA}_{1 \mathrm{c}}$ did not predict any other intermediates, derivatives or oxidative stress-related limiting factors of NO• metabolism.

Regarding the effects of sex, urate and L-citrulline concentrations were lower in female than male participants in both groups, in the fasting and postprandial states (all $p$ values $<0.05$, except for postprandial urate, for which a sex-related effect was non-significant $[p=0.055]$ in people with diabetes) (data not shown). This sexual dimorphism was accompanied by a lower exercise-induced increase in $\triangle \mathrm{THb}$ in female vs male participants in both groups (interaction 'sex' $\times$ 'exercise', $p<0.001$ ) (data not shown).

\section{Discussion}

Unravelling the underlying mechanisms of type 1 diabetesassociated early vascular disorders in response to exercise represents a crucial first step in the design of adequate therapeutics to enhance benefits from physical activity. $\mathrm{NO} \bullet$ is a major vasodilator recruited during exercise and is known to be impacted by the pro-oxidant state in diabetes. However, to our knowledge, this is the first study to explore $\mathrm{NO} \cdot$ metabolism and its oxidative stress-associated limiting factors, as well as a plethora of hormonal/vasoactive moieties, in conjunction with tissue vasoreactivity following exercise in participants with type 1 diabetes vs healthy individuals. Both groups were comparable regarding not only standard demographic criteria but also body composition, physical activity, usual diet and last meal dietary data (including vitamins, polyphenols and nitrate). We found that the previously described [4-6, 17, 18] blunted exercise-induced vasoreactivity in patients with uncomplicated type 1 diabetes vs that in healthy individuals was accompanied by reduced circulating levels of the most abundant water-soluble antioxidant, urate, as well as of L-arginine, the major substrate for eNOS generation of NO• Unexpectedly, exhaustive exercise did not worsen lipid peroxidation or other biomarkers of oxidative stress in patients with type 1 diabetes, and antioxidant resources were mobilised to a comparable extent in both groups.

Canonical NO formation Since the 2000s, the scientific community has shown interest in the impact of type 1 diabetes on ADMA and other L-arginine analogues, sometimes with controversial findings. This discrepancy might be partly explained by analytical issues; only one study reported circulating L-arginine in addition to ADMA in adults with uncomplicated type 1 diabetes vs healthy individuals using an HPLC assay [19]. However, MS (e.g. LC-MS/MS, as used in our study) represents the gold standard for determining L-arginine and dimethylarginines because of its unsurpassed selectivity 

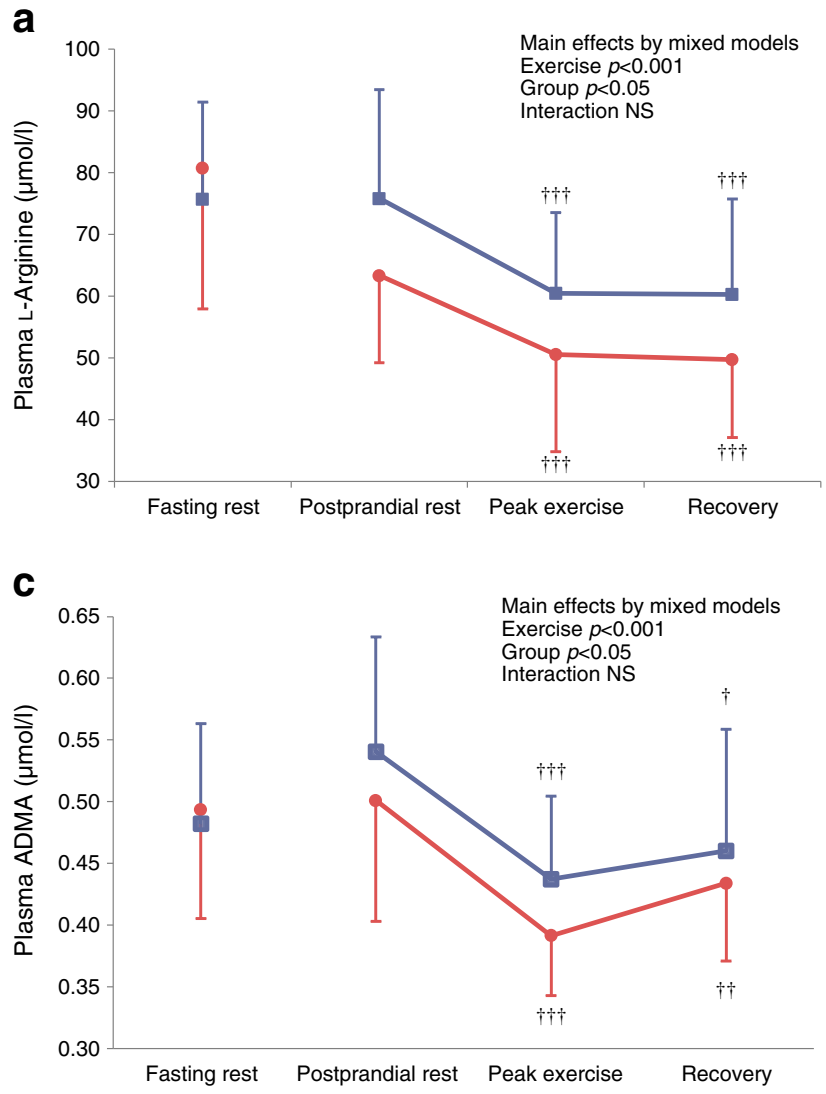

Fig. 1 L-Arginine and analogues at rest (fasting and postprandial) and in response to incremental maximal exercise (postprandial): L-arginine (a); L-citrulline (b); ADMA (c); SDMA (d); data are mean \pm SD. Red circles, participants with type 1 diabetes; blue squares, non-diabetic participants. 'Fasting rest' means during the second visit, in a fasting resting state; 'Postprandial rest', 'Peak exercise' and 'Recovery' mean during the first visit, in a postprandial state, at rest before the exercise, or at peak of

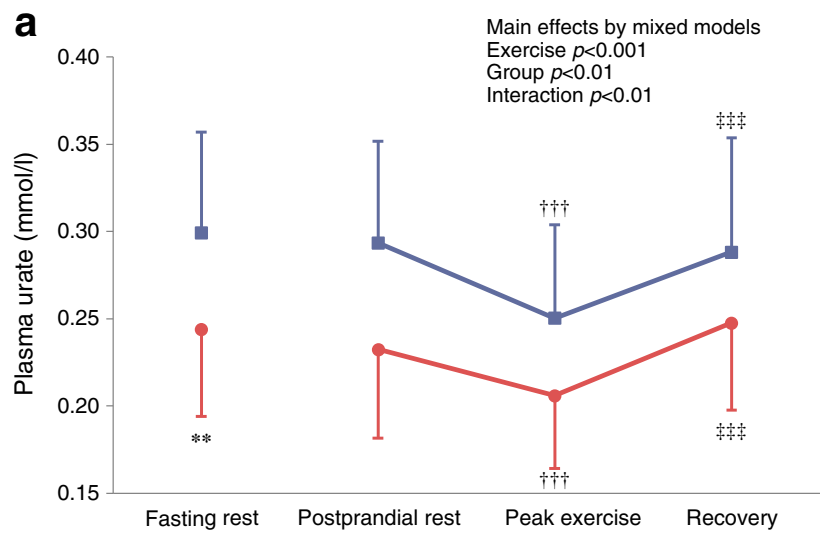

Fig. 2 Plasma urate and total antioxidant status at rest (fasting and postprandial) and in response to incremental maximal exercise (postprandial): plasma urate (a); plasma total antioxidant status (b); data are mean \pm SD. Red circles, participants with type 1 diabetes; blue squares, non-diabetic participants. 'Fasting rest' means during the second visit, in a fasting resting state; 'Postprandial rest', 'Peak exercise' and 'Recovery' mean during the first visit, in a postprandial state, at rest before the exercise, or at peak of exercise or after $15 \mathrm{~min}$ of passive recovery, respectively.
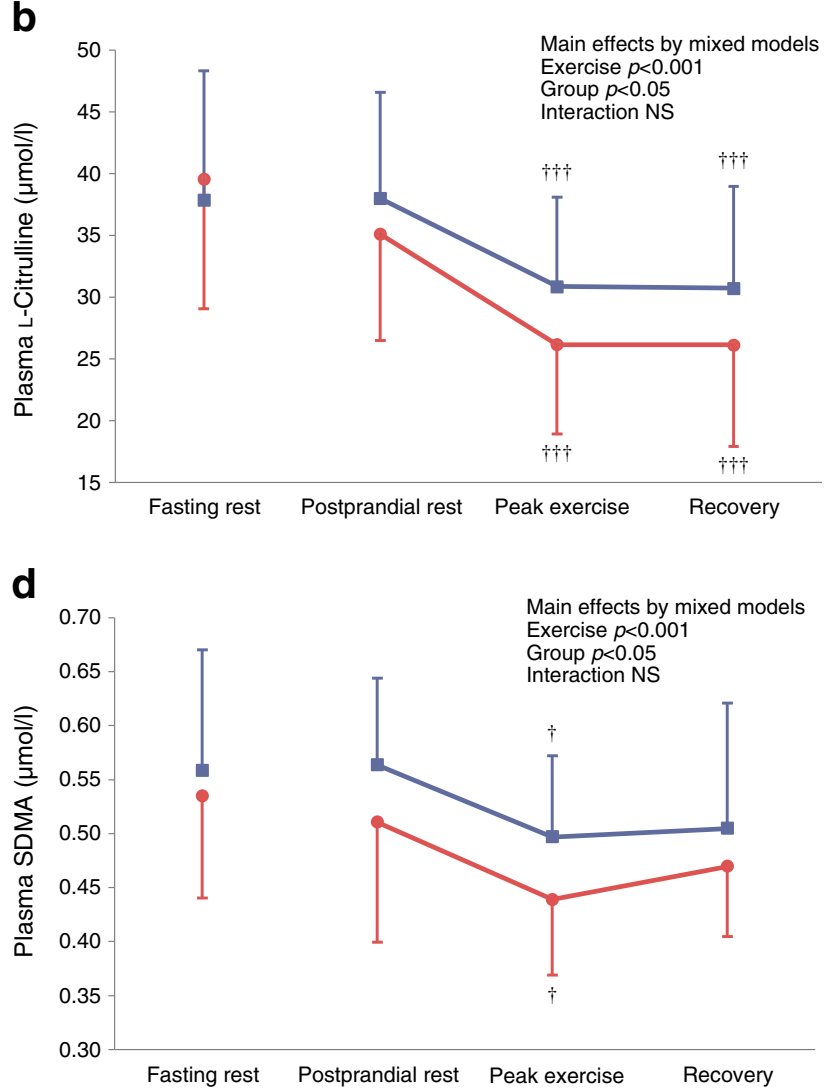

exercise or after $15 \mathrm{~min}$ of passive recovery, respectively. There were no significant intergroup differences in the resting fasting state (Student's unpaired $t$ tests). Main effects from mixed models include $p$ values for an exercise effect (Exercise), a group effect (Group), and an exercise $\times$ group interaction (Interaction). Pairwise post hoc analyses for a time effect: significantly different from those at rest at ${ }^{\dagger} p<0.05$; ${ }^{\dagger \dagger} p<0.01$, or ${ }^{\dagger \dagger} p<0.001$

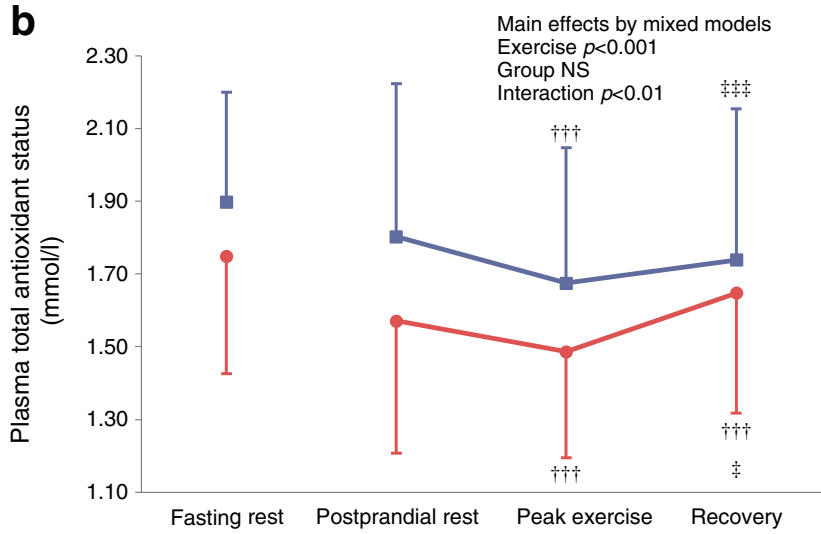

Fasting values: Student's unpaired $t$ tests (urate) or Mann-Whitney $U$ test (total antioxidant status) were performed: significantly different from those of the healthy individuals at $* * p<0.01$. Postprandial values: Main effects from mixed models include $p$ values for an exercise effect (Exercise), a group effect (Group), and an exercise $\times$ group interaction (Interaction). Pairwise post hoc analyses for a time effect: significantly different from those at rest at ${ }^{\dagger \dagger \dagger} p<0.001$; significantly different from those at peak exercise at ${ }^{\star} p<0.05$ or ${ }^{\star \star \star} p p<0.001$ 
[20]. In participants with uncomplicated type 1 diabetes, we found reduced postprandial levels of L-citrulline and L-arginine, plus its methylated analogues ADMA and SDMA. This result is contrary to findings in patients with overt microvascular complications for whom increased L-arginine, ADMA and SDMA levels were reported [21], possibly because of reduced renal clearance. Future investigations are required to identify the mechanisms involved in the drastic reduction in postprandial L-arginine and analogues observed in the earliest stages of type 1 diabetes, as in our study. Glucagon, which was comparable between groups in the postprandial state, does not seem to be involved. In line with a hyperinsulinaemiceuglycaemic clamp study [22], the role of peripheral hyperinsulinaemia, exhibited by the group with type 1 diabetes, can be put forward. High insulin may inhibit protein breakdown in type 1 diabetes [23], and hence indirectly decrease plasma ADMA and SDMA since proteolysis is a key process in their formation [24]. Why L-arginine is also decreased by hyperinsulinaemia deserves further investigation. However, it is noteworthy that, in our study, despite higher insulin levels, levels of L-arginine and its analogues were normal in the fasting state in participants with diabetes. It is then tempting to speculate about a greater insulin resistance of protein metabolism in the fasting state compared with the postprandial state, as already suggested in type 2 diabetes [25]. In addition to hyperinsulinaemia, the role of transient or chronic hyperglycaemia might be discussed, at least with respect to L-arginine levels. There was a strong association between low plasma L-arginine and high $\mathrm{HbA}_{1 \mathrm{c}}$ among the diabetic participants in our study. Bjelakovic et al found that in children with type 1 diabetes, elevated plasma glucose and $\mathrm{HbA}_{1 \mathrm{c}}$ levels correlated with high blood arginase activity; this enzyme competes with NOS for L-arginine [26]. The stimulating role of high glucose on arginase activity and consequently endothelial dysfunction has been confirmed in vitro and in an animal model of diabetes [27]. In addition to a possible deleterious impact of hyperglycaemia on L-arginine availability, the relationship between these two outcomes may also originate from a link occurring in the opposite cause-to-effect direction: it has been shown in diabetic rats that L-arginine inhibits haemoglobin glycation, possibly because of competition exerted by free amino groups of L-arginine during protein glycation [28].

$\mathrm{L}$-arginine is almost the only substrate for eNOS-generating $\mathrm{NO}$, so reduced L-arginine availability in participants with type 1 diabetes in our study might have contributed to the blunted exercise-induced muscle vasoreactivity. Accordingly, in healthy individuals, Penttinen et al [29] showed that systolic BP increased more in response to maximal exercise in individuals with lower baseline plasma L-arginine. Additionally, in an in vitro study, shear stress applied for 40 min increased endothelial NO formation, and this effect was critically dependent on the presence of extracellular Larginine [30]. The lower levels of the endogenous NOS inhibitor, ADMA, in our patients with type 1 diabetes may, at first glance, appear inconsistent with the hypothesis of an impaired eNOS-dependent NO• synthesis. Reduced L-arginine availability, as found in the current study, is known to uncouple eNOS $[31,32]$. In this situation, lower ADMA levels will probably be beneficial by further limiting eNOS uncoupling and associated superoxide production. However, some studies suggest that low ADMA levels are not as beneficial as expected, specifically in the diabetic milieu: a paradoxical inverse relationship between ADMA levels and all-cause mortality has indeed been observed in patients in early stages of diabetes, whereas ADMA is a prognostic marker of all-cause mortality risk in individuals without diabetes [33]. Further studies will be required to determine the actual impact of low ADMA in the context of reduced L-arginine availability in early stages of type 1 diabetes.

In addition to the issue of low L-arginine availability, further studies should also consider the impact of insulin resistance on eNOS-dependent vasoreactivity [2], in view of the higher plasma glucose despite increased circulating insulin in the patients of the current study.

\section{Non-canonical NO formation pathways and other vasoactive} moieties As eNOS-dependent NO• production is known to be attenuated when oxygen is in short supply, alternative sources of NO such as nitrite and $S$-nitrosothiols (part of RXNO) may become more important under exercise-induced tissue hypoxia. As $\mathrm{PaO}_{2}$ was lower in participants with diabetes than in healthy individuals, greater nitrite reduction into bioactive $\mathrm{NO} \cdot$ might have been expected in those with diabetes. However, this does not seem to be the case because we did not find any intergroup difference in serum nitrite. Because only nitrate decreased in response to exercise in both groups, it is possible that circulating nitrate is progressively taken up by working skeletal muscle to be serially reduced to nitrite and $\mathrm{NO} \cdot[34]$.

While nitrite and RXNO were not altered in the individuals with type 1 diabetes (suggesting comparable levels of constitutive eNOS activity [35] in spite of a lower substrate availability), future studies should consider alternative sources of $\mathrm{NO} \cdot$, e.g. nitrosyl haemoglobin. $\mathrm{HbA}_{1 \mathrm{c}}$ has indeed a greater affinity for $\mathrm{NO} \cdot$ compared with non-glycated haemoglobin [36], so that NO• bound to $\mathrm{HbA}_{1 \mathrm{c}}$, transported from regions of high production (i.e. the conduit arteries), may be less readily released downstream [37].

We also measured the arterialised blood concentrations of several ions or gases that, partially independent from the endothelium, can induce smooth vascular cells hyperpolarisation. These moieties did not differ between groups, except for $\mathrm{K}^{+}$, which was lower in diabetic than in non-diabetic individuals. This may be the result of peripheral hyperinsulinaemia because insulin is known to promote $\mathrm{K}^{+}$transfer from the extracellular to the intracellular space, e.g. through the anabolic action of insulin on skeletal muscle $\mathrm{Na}^{+}-\mathrm{K}^{+}$pumps [38]. Although low $\mathrm{K}^{+}$levels are unlikely to be involved in the observed blunted vasoreactivity because extracellular $\mathrm{K}^{+}$ 
operate mainly in the early phase of exercise hyperaemia, the possible deleterious impact of exercise hypokalaemia on muscle excitability and motor function warrants further consideration.

Oxidative stress biomarkers In addition to their possible negative impact on vasoreactivity, reduced L-arginine levels observed in diabetic participants in our study may indirectly increase oxidative stress. Reduced availability of L-arginine has been demonstrated to cause eNOS uncoupling [31, 32] when molecular oxygen serves as the sole electron acceptor and superoxide radical is the main product. The superoxide radical produced by uncoupled eNOS can have detrimental effects on vascular $\mathrm{NO} \cdot$ availability by forming highly reactive peroxynitrite. Mobilisation of antioxidant defences, and particularly urate, to scavenge excess peroxynitrite may be one of the underpinning pathways of significant reduction in plasma urate observed in our participants with type 1 diabetes [39], and still more in female compared with male participants [40]. While high urate levels are conversely associated with cardiovascular events in the advanced stages of diabetes (i.e. in the context of an atherosclerotic milieu) [41], beneficial antioxidant properties of urate are probably fully operative in the earlier stages of the disease. In line with previous research [42], as urate is known to strengthen the antioxidant action of ascorbate with a reduction of lipid peroxidation, the lower urate levels observed in the fasting state in diabetic participants in our study, together with reduced vitamin E/lipids ratio, may contribute to their higher 8 -iso-PGF2 $\alpha$. Formation of these lipid peroxidation products could also have been facilitated by an increased rate of fasting lipolysis (i.e. higher glycerol), a known feature of type 1 diabetes [43].

Of note, while decreased urate levels were probably partly the result of the oxidative burden in our patients, a defect in xanthine oxidase activity cannot be excluded. While admittedly speculative, such a defect could represent another pathway for blunted vasodilatation near maximal exercise, considering that xanthine oxidase is able to produce $\mathrm{NO} \cdot$ from nitrite under hypoxic conditions [34].

Irrespective of the status of $\mathrm{NO} \cdot$ production, oxidative stress was not exacerbated in the participants with diabetes vs healthy participants in the postprandial state. Even more importantly, oxidative stress biomarkers were not raised by exhaustive exercise in either group (serum CML even decreased in both groups). Exercise mobilised antioxidant sources to a similar extent in both groups, with a decrease in the ratio of total free thiols over proteins, a robust marker of extracellular redox status, a decrease in urate as well as in total antioxidant status corrected by urate values, while the activity of erythrocyte glutathione reductase was increased. These changes were likely sufficient to counter exercise-induced free radical production. Thus, complication-free patients with type 1 diabetes are probably no more susceptible to exercise- induced oxidative stress than non-diabetic peers. The findings corroborate the previous few studies on uncomplicated type 1 diabetes showing that oxidative or nitrosative stress response to intense exercise was of comparable magnitude in people both with and without diabetes [44, 45].

A strength of our study is its thorough investigation of NO• metabolism and related oxidative stress together with muscle vasoreactivity in response to exercise. The emerging picture was unexpected and seemingly counterintuitive, with unaltered nitrite and reduced L-arginine, ADMA and SDMA levels. The concomitant hormonal alterations assessed show considerable regulatory complexity around a central node of nitrogen metabolism at the systems level. While NO is known to be a predominant factor of vasodilation, we cannot exclude the involvement of other vasoactive mediators, such as prostanoids, endothelium-derived hyperpolarisation factor, endothelin and angiotensin II. Because L-arginine can inhibit ACE [46], low L-arginine levels might enhance formation of the vasoconstrictor angiotensin II.

In conclusion, our data suggest that individuals with uncomplicated type 1 diabetes display reduced L-arginine availability, possibly in tandem with the anti-proteolytic impact of hyperinsulinaemia. The defect in this essential substrate for enzymatic NO• synthesis may partly contribute to the impaired exercise-induced vasoreactivity. Clinical implications of low Larginine levels are probably not limited to vasodilatation since $\mathrm{NO} \cdot$ is also essential for regulation of important processes in skeletal muscle, including mitochondrial respiration, glucose uptake and force production. Thus, future work testing L-citrulline supplementation might be promising (L-citrulline raises circulating $\mathrm{L}$-arginine without being eliminated from the intestine as L-arginine is) [47]. Plasma urate, the most abundant water-soluble antioxidant, was also reduced in participants with type 1 diabetes, which may contribute to endothelial dysfunction [48]. However, these low urate concentrations unexpectedly did not translate into an exacerbated oxidative stress in response to exhaustive exercise. This encouraging result suggests that individuals with as yet uncomplicated diabetes can practice physical exercise, even if intense, without risking more oxidative damages.

Acknowledgements The authors would like to thank A. Descatoire, C. Fermon, M. Lepeut (CETRADIMN, Roubaix Regional Hospital), F. Baudoux, and A. Vambergue (Diabetes Department, Lille University Hospital) for helping with the recruitment of patients; P. Hincker, P. Rasoamanana, I. Rougeaux, N. Waucquier and D. Deplanque (Clinical Investigation Center, Lille University Hospital), F. Dehaut, J. Aucouturier, FX. Gamelin, F. Prieur, P. Mucci, G. Baquet, C. Parent, J. Plantec (ULR 7369 - URePSSS - Unité de Recherche Pluridisciplinaire Sport Santé Société, University of Lille), A. Watry, M Nunes-Leclercq (Diabetes Department, Lille University Hospital), E. Devemy, M. Dominikowski, K. Mimeche and M. Leclercq (Pulmonary Function Testing Department, Lille University Hospital) for laboratory assistance; A.F Dessein, N. Rouaix, P. Pigny, M.J. Lepoutre, M. Michiels, and L. Sapyn (Laboratory of Biochemistry and Endocrinology, Lille University Hospital), B. Callens (Univ. Lille, CHU Lille, EA 7365 - GRITA Groupe de Recherche sur les formes Injectables et les Technologies 
Associées, F-59000 Lille, France), M. Minnion (Clinical and Experimental Sciences, Faculty of Medicine, University of Southampton), and N. Grossin (Univ. Lille, Inserm, CHU Lille) for blood analyses; C. Lepretre (Clinical Research Direction, Lille University Hospital), J. Lepoutre, J. Naturel, C. Dumoitier, A. Meresse (University of Lille) for administrative management; S. Platt and B. Heyman for revising the English; A. Bertrand (Statistical Methodology and Computing Service (SMCS), UCLouvain) for statistical analyses. We also thank Linde Homecare France for their kind donation via which E. Lespagnol was recruited as a postdoctoral researcher.

Data availability The datasets generated during and/or analysed during the current study are available from the corresponding author on reasonable request.

Funding This study was supported by grants obtained by EH and PF from SFD (Société Francophone du Diabète: grant ALFEDIAM-Roche Diagnostics 2009), from the interregional hospital programme of clinical research (PHRC, 2010), and from the North of France Region (Programme Emergent, 2013). These study funders were not involved in the design of the study; the collection, analysis, and interpretation of data; or writing the report; and did not impose any restrictions regarding the publication of the report.

Duality of interest No potential conflict of interest relevant to this article to report.

Authors' relationships and activities The authors declare that there are no relationships or activities that might bias, or be perceived to bias, their work.

Contribution statement EH designed the experiments and contributed to their performance, analysed data and wrote the manuscript. EL analysed data and helped with writing the manuscript. PF contributed to the conception of the experiments, patient recruitment and review of the manuscript. MF contributed to the performance of the experiments and helped with writing the manuscript. ST performed the experiments, helped with data analysis, designed the figures and the graphical abstract, and reviewed the manuscript. BOF, FZ, RM, PM, AD, IK, MP-C and EB contributed to the performance of the experiments and review of the manuscript. SB contributed to the conception of the experiments and review of the manuscript. JB contributed to data analyses and review of the manuscript. All authors approved the final version of the manuscript. $\mathrm{EH}$ is the guarantor of this work and, as such, had full access to all data in the study and takes responsibility for the integrity of the data and the accuracy of the data analysis.

\section{References}

1. Sibal L, Aldibbiat A, Agarwal SC et al (2009) Circulating endothelial progenitor cells, endothelial function, carotid intima-media thickness and circulating markers of endothelial dysfunction in people with type 1 diabetes without macrovascular disease or microalbuminuria. Diabetologia 52(8):1464-1473. https://doi.org/ 10.1007/s00125-009-1401-0

2. Rask-Madsen C, King GL (2007) Mechanisms of disease: endothelial dysfunction in insulin resistance and diabetes. Nat Clin Pract Endocrinol Metab 3(1):46-56. https://doi.org/10.1038/ ncpendmet0366

3. Lespagnol E, Dauchet L, Pawlak-Chaouch M et al (2020) Early endothelial dysfunction in type 1 diabetes is accompanied by an impairment of vascular smooth muscle function: a meta-analysis.
Front Endocrinol (Lausanne) 11:203. https://doi.org/10.3389/ fendo.2020.00203

4. Heyman E, Daussin F, Wieczorek V et al (2020) Muscle oxygen supply and use in type 1 diabetes, from ambient air to the mitochondrial respiratory chain: is there a limiting step? Diabetes Care 43(1): 209-218. https://doi.org/10.2337/dc19-1125

5. Rissanen AP, Tikkanen HO, Koponen AS, Aho JM, Peltonen JE (2015) Central and peripheral cardiovascular impairments limit VO(2peak) in type 1 diabetes. Med Sci Sports Exerc 47(2):223230. https://doi.org/10.1249/MSS.0000000000000419

6. Tagougui S, Leclair E, Fontaine P et al (2015) Muscle oxygen supply impairment during exercise in poorly controlled type 1 diabetes. Med Sci Sports Exerc 47(2):231-239. https://doi.org/10. 1249/MSS.0000000000000424

7. Whelton SP, McAuley PA, Dardari Z et al (2020) Association of BMI, fitness, and mortality in patients with diabetes: evaluating the obesity paradox in the Henry Ford Exercise Testing Project (FIT Project) Cohort. Diabetes Care 43(3):677-682. https://doi.org/10. 2337/dc19-1673

8. Hickner RC, Fisher JS, Ehsani AA, Kohrt WM (1997) Role of nitric oxide in skeletal muscle blood flow at rest and during dynamic exercise in humans. Am J Phys 273(1 Pt 2):H405-H410. https:// doi.org/10.1152/ajpheart.1997.273.1.H405

9. Kelm M, Preik-Steinhoff H, Preik M, Strauer BE (1999) Serum nitrite sensitively reflects endothelial NO formation in human forearm vasculature: evidence for biochemical assessment of the endothelial L-arginine-NO pathway. Cardiovasc Res 41(3):765-772. https://doi.org/10.1016/s0008-6363(98)00259-4

10. Fayh AP, Krause M, Rodrigues-Krause J et al (2013) Effects of Larginine supplementation on blood flow, oxidative stress status and exercise responses in young adults with uncomplicated type I diabetes. Eur J Nutr 52(3):975-983. https://doi.org/10.1007/s00394-0120404-7

11. Fujii N, Dervis S, Sigal RJ, Kenny GP (2016) Type 1 diabetes modulates cyclooxygenase- and nitric oxide-dependent mechanisms governing sweating but not cutaneous vasodilation during exercise in the heat. Am J Physiol Regul Integr Comp Physiol 311(6):R1076-R1084. https://doi.org/10.1152/ajpregu.00376.2016

12. Kriska AM, Knowler WC, LaPorte RE et al (1990) Development of questionnaire to examine relationship of physical activity and diabetes in Pima Indians. Diabetes Care 13(4):401-411. https:// doi.org/10.2337/diacare.13.4.401

13. Barstow TJ (2019) CORP: Understanding near infrared spectroscopy (NIRS) and its application to skeletal muscle research. J Appl Physiol (1985) 126(5):1360-1376. https://doi.org/10.1152/ japplphysiol.00166.2018

14. Franklin VL, Khan F, Kennedy G, Belch JJ, Greene SA (2008) Intensive insulin therapy improves endothelial function and microvascular reactivity in young people with type 1 diabetes. Diabetologia 51(2):353-360. https://doi.org/10.1007/s00125-0070870-2

15. Boden G, Rezvani I, Owen OE (1984) Effects of glucagon on plasma amino acids. J Clin Invest 73(3):785-793. https://doi.org/ 10.1172/JCI111272

16. Van Beaumont W (1972) Evaluation of hemoconcentration from hematocrit measurements. J Appl Physiol 32(5):712-713. https:// doi.org/10.1152/jappl.1972.32.5.712

17. Matthys D, Craen M, De Wolf D, Vande Walle J, Verhaaren H (1996) Reduced decrease of peripheral vascular resistance during exercise in young type I diabetic patients. Diabetes Care 19(11): 1286-1288. https://doi.org/10.2337/diacare.19.11.1286

18. Zwingli G, Yerly J, Mivelaz Y et al (2020) Non-invasive assessment of coronary endothelial function in children and adolescents with type 1 diabetes mellitus using isometric handgrip exerciseMRI: a feasibility study. PLoS One 15(2):e0228569. https://doi. org/10.1371/journal.pone.0228569 
19. Altinova AE, Arslan M, Sepici-Dincel A, Akturk M, Altan N, Toruner FB (2007) Uncomplicated type 1 diabetes is associated with increased asymmetric dimethylarginine concentrations. J Clin Endocrinol Metab 92(5):1881-1885. https://doi.org/10.1210/ jc.2006-2643

20. Schwedhelm E (2005) Quantification of ADMA: analytical approaches. Vasc Med 10(Suppl 1):S89-S95. https://doi.org/10. 1177/1358836X0501000113

21. Abhary S, Kasmeridis N, Burdon KP et al (2009) Diabetic retinopathy is associated with elevated serum asymmetric and symmetric dimethylarginines. Diabetes Care 32(11):2084-2086. https://doi. org/10.2337/dc09-0816

22. Marcovecchio ML, Widmer B, Dunger DB, Dalton RN (2008) Effect of acute variations of insulin and glucose on plasma concentrations of asymmetric dimethylarginine in young people with type 1 diabetes. Clin Sci (Lond) 115(12):361-369. https://doi.org/10. 1042/CS20080079

23. Bennet WM, Connacher AA, Jung RT, Stehle P, Rennie MJ (1991) Effects of insulin and amino acids on leg protein turnover in IDDM patients. Diabetes 40(4):499-508. https://doi.org/10.2337/diab.40. 4.499

24. Su Y, Block ER (1996) Acute hypoxia increases intracellular Larginine content in cultured porcine pulmonary artery endothelial cells. J Cell Physiol 167(2):349-353. https://doi.org/10.1002/(SICI) 1097-4652(199605)167:2<349::AID-JCP20>3.0.CO;2-1

25. Bassil M, Marliss EB, Morais JA, Pereira S, Chevalier S, Gougeon R (2011) Postprandial hyperaminoacidaemia overcomes insulin resistance of protein anabolism in men with type 2 diabetes. Diabetologia 54(3):648-656. https://doi.org/10.1007/s00125-0101980-9

26. Bjelakovic G, Sokolovic D, Ljiljana S et al (2009) Arginase activity and magnesium levels in blood of children with diabetes mellitus. J Basic Clin Physiol Pharmacol 20(4):319-334. https://doi.org/10. 1515/jbcpp.2009.20.4.319

27. Romero MJ, Platt DH, Tawfik HE et al (2008) Diabetes-induced coronary vascular dysfunction involves increased arginase activity. Circ Res 102(1):95-102. https://doi.org/10.1161/CIRCRESAHA. 107.155028

28. Mendez JD, Balderas FL (2006) Inhibition by L-arginine and spermidine of hemoglobin glycation and lipid peroxidation in rats with induced diabetes. Biomed Pharmacother 60(1):26-31. https:// doi.org/10.1016/j.biopha.2005.08.004

29. Penttinen J, Toyry J, Eerikainen S, Litmanen H, Rinkinen T, Lansimies E (1998) Does plasma L-arginine level play a role in regulation of blood pressure during exercise and autonomic nervous function? Clin Physiol 18(6):539-543. https://doi.org/10. 1046/j.1365-2281.1998.00132.x

30. Posch K, Schmidt K, Graier WF (1999) Selective stimulation of Larginine uptake contributes to shear stress-induced formation of nitric oxide. Life Sci 64(8):663-670. https://doi.org/10.1016/ s0024-3205(98)00608-0

31. Kim JH, Bugaj LJ, Oh YJ et al (2009) Arginase inhibition restores NOS coupling and reverses endothelial dysfunction and vascular stiffness in old rats. J Appl Physiol (1985) 107(4):1249-1257. https://doi.org/10.1152/japplphysiol.91393.2008

32. Stuehr D, Pou S, Rosen GM (2001) Oxygen reduction by nitricoxide synthases. J Biol Chem 276(18):14533-14536. https://doi. org/10.1074/jbc.R100011200

33. Anderssohn M, Schwedhelm E, Luneburg N, Vasan RS, Boger RH (2010) Asymmetric dimethylarginine as a mediator of vascular dysfunction and a marker of cardiovascular disease and mortality: An intriguing interaction with diabetes mellitus. Diab Vasc Dis Res 7(2):105-118. https://doi.org/10.1177/1479164110366053

34. Piknova B, Park JW, Kwan Jeff Lam K, Schechter AN (2016) Nitrate as a source of nitrite and nitric oxide during exercise hyperemia in rat skeletal muscle. Nitric Oxide 55-56:54-61. https://doi.org/10.1016/j.niox.2016.03.005

35. Kleinbongard P, Dejam A, Lauer T et al (2003) Plasma nitrite reflects constitutive nitric oxide synthase activity in mammals. Free Radic Biol Med 35(7):790-796. https://doi.org/10.1016/ s0891-5849(03)00406-4

36. James PE, Lang D, Tufnell-Barret T, Milsom AB, Frenneaux MP (2004) Vasorelaxation by red blood cells and impairment in diabetes: Reduced nitric oxide and oxygen delivery by glycated hemoglobin. Circ Res 94(7):976-983. https://doi.org/10.1161/01.RES. 0000122044.21787 .01

37. Milsom AB, Jones CJ, Goodfellow J, Frenneaux MP, Peters JR, James PE (2002) Abnormal metabolic fate of nitric oxide in type I diabetes mellitus. Diabetologia 45(11):1515-1522. https://doi.org/ 10.1007/s00125-002-0956-9

38. Schmidt TA, Hasselbalch S, Farrell PA, Vestergaard H, Kjeldsen K (1994) Human and rodent muscle $\mathrm{Na}(+)-\mathrm{K}(+)$-ATPase in diabetes related to insulin, starvation, and training. J Appl Physiol (1985) 76(5):2140-2146. https://doi.org/10.1152/jappl.1994.76.5.2140

39. Pitocco D, Di Stasio E, Romitelli F et al (2008) Hypouricemia linked to an overproduction of nitric oxide is an early marker of oxidative stress in female subjects with type 1 diabetes. Diabetes Metab Res Rev 24(4):318-323. https://doi.org/10.1002/dmrr.814

40. Marra G, Cotroneo P, Pitocco D et al (2002) Early increase of oxidative stress and reduced antioxidant defenses in patients with uncomplicated type 1 diabetes: a case for gender difference. Diabetes Care 25(2):370-375. https://doi.org/10.2337/diacare.25. 2.370

41. Pilemann-Lyberg S, Hansen TW, Tofte N et al (2019) Uric acid is an independent risk factor for decline in kidney function, cardiovascular events, and mortality in patients with type 1 diabetes. Diabetes Care 42(6):1088-1094. https://doi.org/10.2337/dc18-2173

42. Francescato MP, Stel G, Geat M, Cauci S (2014) Oxidative stress in patients with type 1 diabetes mellitus: is it affected by a single bout of prolonged exercise? PLoS One 9(6):e99062. https://doi.org/10. 1371/journal.pone.0099062

43. Hagstrom-Toft E, Bolinder J, Ungerstedt U, Arner P (1997) A circadian rhythm in lipid mobilization which is altered in IDDM. Diabetologia 40(9):1070-1078. https://doi.org/10.1007/ s001250050789

44. Laaksonen DE, Atalay M, Niskanen L, Uusitupa M, Hanninen O, Sen CK (1996) Increased resting and exercise-induced oxidative stress in young IDDM men. Diabetes Care 19(6):569-574. https:// doi.org/10.2337/diacare.19.6.569

45. Rosa JS, Oliver SR, Flores RL et al (2011) Altered inflammatory, oxidative, and metabolic responses to exercise in pediatric obesity and type 1 diabetes. Pediatr Diabetes 12(5):464-472. https://doi. org/10.1111/j.1399-5448.2010.00724.x

46. Higashi $\mathrm{Y}$, Oshima $\mathrm{T}$, Ono $\mathrm{N}$ et al (1995) Intravenous administration of L-arginine inhibits angiotensin-converting enzyme in humans. J Clin Endocrinol Metab 80(7):2198-2202. https://doi. org/10.1210/jcem.80.7.7608279

47. McCarthy O, Moser O, Eckstein ML, Bain SC, Pitt J, Bracken R (2019) Supplementary nitric oxide donors and exercise as potential means to improve vascular health in people with type 1 diabetes: yes to no? Nutrients 11(7):1571. https://doi.org/10.3390/ nu11071571

48. Waring WS, McKnight JA, Webb DJ, Maxwell SR (2006) Uric acid restores endothelial function in patients with type 1 diabetes and regular smokers. Diabetes 55(11):3127-3132. https://doi.org/ $10.2337 / \mathrm{db} 06-0283$

Publisher's note Springer Nature remains neutral with regard to jurisdictional claims in published maps and institutional affiliations. 